\title{
HRTEM Imaging of Atoms at Sub-Ångström Resolution
}

\author{
Michael A. O'Keefe*, Lawrence F. Allard** and Douglas A. Blom** \\ *Materials Sciences Division, Lawrence Berkeley National Laboratory, \\ 1 Cyclotron Road, Berkeley, CA 94720, USA. \\ ** Metals and Ceramics Division, Oak Ridge National Laboratory, \\ Oak Ridge, TN 37831-6064, USA
}

Dedicated to the memory of the late John M. Cowley.

$\begin{array}{lc}\text { Corresponding Author: } & \begin{array}{c}\text { Michael A. O'Keefe, } \\ \text { maok@lbl.gov } \\ \text { email }\end{array} \\ \text { phone } & 510-486-4610 \\ \text { fax: } & 510-486-5530 \\ \text { mailing address: } & \text { LBNL MS 2R200, } 1 \text { Cyclotron Road, Berkeley, CA 94720, USA }\end{array}$

Running head: $\quad$ Imaging Atoms at Sub-Ångström Resolution

Keywords: $\quad$ Sub-Ångström, atomic-resolution, high-resolution electron microscopy, focalseries reconstruction, exit-surface wave, software aberration correction.

\begin{abstract}
This document was prepared as an account of work sponsored by the United States Government. While this document is believed to contain correct information, neither the United States Government nor any agency thereof, nor The Regents of the University of California, nor any of their employees, makes any warranty, express or implied, or assumes any legal responsibility for the accuracy, completeness, or usefulness of any information, apparatus, product, or process disclosed, or represents that its use would not infringe privately owned rights. Reference herein to any specific commercial product, process, or service by its trade name, trademark, manufacturer, or otherwise, does not necessarily constitute or imply its endorsement, recommendation, or favoring by the United States Government or any agency thereof, or The Regents of the University of California. The views and opinions of authors expressed herein do not necessarily state or reflect those of the United States Government or any agency thereof, or The Regents of the University of California.
\end{abstract}

Ernest Orlando Lawrence Berkeley National Laboratory is an equal opportunity employer. 


\begin{abstract}
John Cowley and his group at Arizona State University pioneered the use of transmission electron microscopy (TEM) for high-resolution imaging. Images were achieved three decades ago showing the crystal unit cell content at better than $4 \AA$ resolution. This achievement enabled researchers to pinpoint the positions of heavy atom columns within the unit cell. Lighter atoms appear as resolution is improved to sub-Ångström levels. Currently, advanced microscopes can image the columns of the light atoms (carbon, oxygen, nitrogen) that are present in many complex structures, and even the lithium atoms present in some battery materials. SubÅngström imaging, initially achieved by focal-series reconstruction of the specimen exit surface wave, will become commonplace for next-generation electron microscopes with $\mathrm{C}_{\mathrm{S}}$-corrected lenses and monochromated electron beams. Resolution can be quantified in terms of peak separation and inter-peak minimum, but the limits imposed on the attainable resolution by the properties of the microscope specimen need to be considered. At extreme resolution the "size" of atoms can mean that they will not be resolved even when spaced farther apart than the resolution of the microscope.
\end{abstract}




\section{Introduction}

Modern high-resolution transmission electron microscopes (HRTEMs) can produce images with discrete peaks corresponding to atoms or to columns of atoms viewed end-on. For the materials scientist, the important property of the image is to show where atoms (or atom columns) are positioned relative to one another. Ability to determine if an image peak represents one single atom (or atom column) instead of several depends on the resolution of the HRTEM.

Resolution was first defined in terms of the classic case of separation of adjacent objects [1]. Considerations of noise and coherence in detected images require some modification of this simple definition (for a thorough discussion see [2]). Nevertheless, HRTEM resolution requires the ability to produce images showing distinct separation of discrete objects (atoms or columns of atoms) and the determination of their relative positions. Higher resolution not only allows the identification of closer objects, the finer sampling improves the detection of weaker signals, thus making possible the imaging of lighter atoms in the presence of heavier ones. Over the past three decades, improvements in transmission electron microscope (TEM) resolution have enabled materials scientists to move from general observations of large-scale defects (grain boundaries, dislocations) to studies of details within the crystal unit cell. Recent advances have allowed the imaging of all atoms within the unit cell, as well as the study of non-periodic defects with closely separated projected atom positions.

Early studies at $7 \AA$ resolution revealed the positions of crystallographic shear planes within unit cells of transition metal oxides [3]. Positions of metal-oxide octahedra appeared at $3.5 \AA$ resolution [4]. Individual metal-atom columns became accessible in alloys at 1.8-2.0Å resolution [5], and in silicates at $1.6 \AA$ resolution [6]. Development of software for simulation of HREM images from structural models explained the images and confirmed their interpretations [7-14]. 
For uncorrected microscopes, resolution limits are set by phase changes imposed by spherical aberration [15], and remain at $1 \AA$ for high-voltage ( $\geq 1 \mathrm{MV})$ TEMs and $1.5 \AA$ for mid-voltage TEMs. Methods to overcome the "Scherzer limit" by correcting for phase changes include focalseries reconstruction of the electron wave at the specimen exit surface [16-17], holography [18], and hardware correction of spherical aberration [19].

\section{Imaging Atoms in the Electron Microscope}

\section{a) Image Formation}

The process of image formation in the HRTEM is well understood [20-21]. Image intensity is the square of the complex image amplitude, or electron wave, at the image plane. Its Fourier transform, the image intensity spectrum, is the auto-correlation of the image amplitude spectrum,

$$
\mathrm{I}(\mathbf{u})=\sum_{\mathbf{u}^{\prime}} \Psi_{\mathbf{E}}\left(\mathbf{u}^{\prime}\right) \cdot \exp \left\{-\mathrm{i} \chi\left(\mathbf{u}^{\prime}\right)\right\} \cdot \Psi_{\mathbf{E}}^{*}\left(\mathbf{u}^{\prime}-\mathbf{u}\right) \cdot \exp \left\{+\mathrm{i} \chi\left(\mathbf{u}^{\prime}-\mathbf{u}\right)\right\}
$$

where $\Psi_{\mathbf{E}}(\mathbf{u})$ is the $\mathbf{u}$ th Fourier component of the exit-surface electron wave that is modulated by a function $\chi(\mathbf{u})$ that describes phase changes from the objective lens.

For a thin specimen (or a thicker one not precisely oriented down a zone-axis), most contributions to the image intensity spectrum come from interference of beams with the central (000) beam. Non-000 interference ("second order" or "non-linear" interference) contributes only weakly and may be ignored [22]. Under the condition of linear interference only one pair of diffracted beams contributes to each spatial frequency in the image intensity spectrum [21].

$$
\mathrm{I}_{\mathbf{L}}(\mathbf{u})=\Psi_{\mathbf{E}}(\mathbf{u}) \cdot \exp [-\mathrm{i} \chi(\mathbf{u})]+\Psi_{\mathbf{E}}^{*}(-\mathbf{u}) \cdot \exp [+\mathrm{i} \chi(-\mathbf{u})]
$$

where $\Psi(\mathbf{0})$ has been normalized out, since it has a weight that is close to unity for a weakly scattering specimen and is common to all linear interference terms. 
For elastic scattering the specimen behaves as a "phase object" with information on (projected) specimen potential $\phi_{\mathrm{p}}(\mathbf{x})$ and specimen thickness $\mathrm{H}$ carried in the phase of the electron wave [23]

$$
\psi_{\mathbf{E}}(\mathbf{x})=\exp \left\{-\mathrm{i} \sigma \phi_{\mathrm{p}}(\mathbf{x}) \mathrm{H}\right\}
$$

where $\sigma$ is the interaction coefficient, $\phi_{\mathrm{p}}(\mathbf{x})$ is the specimen potential projected in the incident electron beam direction, and $\mathrm{H}$ is specimen thickness. In a weakly scattering specimen, the majority of elastically scattered electrons will undergo kinematic (single) scattering and the direct-space electron wave at the specimen exit-surface of this "weak phase object" will be

$$
\psi_{\mathbf{E}}(\mathbf{x})=1-\mathrm{i} \sigma \phi_{\mathrm{p}}(\mathbf{x}) \mathrm{H}
$$

The linear image intensity spectrum (eqn.2) becomes

$$
\mathrm{I}_{\mathbf{L}}(\mathbf{u})=\delta(\mathbf{u})+2 \sigma \mathrm{V}(\mathbf{u}) \mathrm{H} \sin \chi(\mathbf{u})
$$

where $\{\exp [\mathrm{i} \chi(\mathbf{u})]-\exp [-\mathrm{i} \chi(-\mathbf{u})]\}=2 \mathrm{i} \sin \chi(\mathbf{u})$ for a round lens, $\mathrm{V}(\mathbf{u})$ is the (complex) Fourier component of the (real) projected potential $\phi_{\mathrm{p}}(\mathbf{x})$, and $\mathrm{V}^{*}(-\mathbf{u})=\mathrm{V}(\mathbf{u})$.

Equation (5) shows that the magnitude of the uth term in the image intensity spectrum is proportional to $\mathrm{V}(\mathbf{u})$, the uth Fourier coefficient of the projected potential, and to $\sin \chi(\mathbf{u})$, the value of the phase-contrast transfer function (CTF) at the corresponding value of $|\mathbf{u}|$.

\section{b) Image Resolution}

The phase change due to objective lens defocus $\varepsilon$ and spherical aberration coefficient $\mathrm{C}_{\mathrm{S}}$ at an electron wavelength $\lambda$ is

$$
\chi(\mathbf{u})=\pi \varepsilon \lambda|\mathbf{u}|^{2}+\pi \mathrm{C}_{\mathrm{S}} \lambda^{3}|\mathbf{u}|^{4} / 2
$$


The resolution of the electron microscope corresponds to the highest spatial frequency that is transferred into the image intensity spectrum with the same sign as all lower contributing frequencies. When $\chi(\mathbf{u})$ is set equal to zero, the cutoff (zero-crossing) frequency is given by

$$
|\mathbf{u}|_{S}=\sqrt{ }\left\{-2 \varepsilon /\left(\mathrm{C}_{\mathrm{S}} \lambda^{2}\right)\right\}
$$

At the Scherzer [15] optimum defocus of $\varepsilon=-\sqrt{ }\left(C_{S} \lambda\right)$ the cutoff frequency is $|\mathbf{u}|=\sqrt{ } 2 C_{S}^{-1 / 4} \lambda^{-3 / 4}$ $\left(1.414 \mathrm{C}_{\mathrm{S}}^{-1 / 4} \lambda^{-3 / 4}\right)$. Careful comparisons of simulated "weak-phase" images with simulated highresolution lattice image (SHRLI) images [21] showed that the cutoff frequency could be extended to $6{ }^{1 / 4} \mathrm{C}_{\mathrm{S}}{ }^{-1 / 4} \lambda^{-3 / 4}\left(1.565 \mathrm{C}_{\mathrm{S}}{ }^{-1 / 4} \lambda^{-3 / 4}\right)$ by choosing a defocus of $\varepsilon=-\sqrt{ }\left(3 \mathrm{C}_{\mathrm{S}} \lambda / 2\right)$. The Scherzer resolution of the microscope is then

$$
\mathrm{d}_{\mathrm{S}}=0.64 \mathrm{C}_{\mathrm{S}}{ }^{1 / 4} \lambda^{3 / 4}
$$

At Scherzer defocus $\sin \chi(\mathbf{u})$ is approximately equal to -1 . Each term in the intensity spectrum is proportional to (the negative of) the corresponding Fourier coefficient of the projected potential. Inverse Fourier transformation of the intensity spectrum (eqn.5) yields image intensity proportional to the negative magnitude of the projected potential. Peaks in the potential at the atom positions will produce dips in the image intensity; the image will show "black atoms".

$$
\mathrm{I}_{\mathbf{L}}(\mathbf{x})=1-2 \sigma \phi_{\mathrm{p}}(\mathbf{x}) \mathrm{H}
$$

\section{c) Information Limit}

The incident electron beam has partial spatial and temporal coherence that impose limits on the highest spatial frequency that can be transferred from the exit-surface wave to the image. Partial temporal coherence manifests as spread of focus, and partial spatial coherence as incident beam 
convergence [13]. These factors attenuate the microscope CTF with 'damping envelope' functions [24-25].

The CTF damping envelope function for temporal incoherence has the form

$$
\mathrm{E}_{\Delta}(\mathbf{u})=\exp \left\{-1 / 2 \pi^{2} \lambda^{2} \Delta^{2}|\mathbf{u}|^{4}\right\}
$$

where $\Delta$ is the standard deviation of a Gaussian spread-of-focus. Transfer falls to $\exp (-2)$ or $13.5 \%$ at $|\mathbf{u}|_{\Delta}=(\pi \lambda \Delta / 2)^{-1 / 2}$, leading to an absolute information limit for the microscope of

$$
\mathrm{d}_{\Delta}=1 /|\mathbf{u}|_{\Delta}=\sqrt{ }(\pi \lambda \Delta / 2)
$$

The $\Delta$ parameter includes changes in microscope focus over image acquisition time from lens current ripple and high-voltage noise and ripple, as well as incident beam energy spread due to intrinsic gun spread and the Boersch contribution [26]. Then

$$
\Delta=\mathrm{C}_{\mathrm{C}} \sqrt{ }\left[4 \sigma^{2}(\mathrm{I}) / \mathrm{I}^{2}+\sigma^{2}(\mathrm{E}) / \mathrm{E}^{2}\right]
$$

where $C_{C}$ is the chromatic aberration coefficient for the objective lens, $\sigma(I) / I$ is the fractional rms ripple in lens current, and $\sigma(\mathrm{E}) / \mathrm{E}$ is the rms energy spread in the electron beam (including highvoltage noise and ripple) as a fraction of the total beam energy.

\section{d) Spatial Coherence}

The damping envelope function for a Gaussian-weighted incident beam convergence is

$$
\mathrm{E}_{\alpha}(\mathbf{u})=\exp \left\{-\pi^{2} \alpha^{2}\left(\varepsilon+\lambda^{2} \mathrm{C}_{\mathrm{S}} \mathbf{u}^{2}\right)^{2} \mathbf{u}^{2}\right\}
$$

where $\alpha$ is the standard deviation of a Gaussian over the convergent cone, equal to 0.77 times the measured semi-angle [27]. Like spread of focus, convergence limits information transfer at high spatial frequencies. However, spread of focus imposes an absolute limit for the microscope whereas the convergence limit can be changed by changing defocus [21]. 


\section{e) Phase}

The complex functions that describe electron microscope imaging have phases, and these phases must remain undistorted if images are to show atom positions correctly. Information on atom positions is carried by the phase of the real-space electron wave exiting the specimen (the ESW). Phases of reciprocal-space components of real-space functions can be modified by multiple scattering inside the specimen and by the phase changes of the lens forming the image. It is important that clear distinctions be maintained about the roles of these phases in the description of electron scattering and their effects on the microscope image.

Structure Factor Phase: The incoming electron wave is modulated by the specimen potential $\phi_{\mathrm{p}}(\mathbf{x})$ described in Fourier space by a set of structure factors $\mathrm{V}(\mathbf{u})$ with magnitudes that give relative weights to spacings in the potential distribution, and phases that give their positions (a phase change in reciprocal space is a shift in real space). In structure determination, the difficulty of measurement of these phases is known as "the phase problem".

Electron Wave Phase: The real-space electron wave exiting the specimen (the ESW) carries information about the specimen in the form of a spatial distribution of phase changes. The relative phase of the wave depends linearly on specimen thickness (the number of atoms stacked in the beam direction). In a crystal, the spatial distribution of the ESW phase shows the positions of atom columns and even the relative scattering power of the types of atoms in each column.

Objective Lens Phase: The ESW passes through the objective lens. Diffracted beams (the Fourier components of the ESW) suffer phase changes as they are brought together by the lens to interfere and form the image intensity spectrum. The lens phase changes depend on many lens 
parameters and aberrations, primarily defocus and spherical aberration, and are described by the lens phase function $\chi(\mathbf{u})$.

Phase Interactions and Scherzer Imaging: At Scherzer defocus the lens transfer function adds approximately the same phase change to the ESW within a band of spatial frequencies out to the resolution limit of the microscope (CTF transfer is also maximized and approximately constant to the same limit). The relative phase of each component of the image-plane wave is the same as that at the corresponding spatial frequency in the ESW. Additionally, with the spatial distribution of the ESW phase properly mapped into the phase of the image-plane wave, there is no "phase problem" for the structure factors, and the image will display the projected potential of the specimen to the resolution limit of the microscope (eqn.8). However, incorrect lens phase changes on higher-frequency image components beyond the Scherzer cutoff will not preserve the relative phase of the ESW. The ESW will be mapped into the image with phase distortions, and the image will not show the positions and relative weights of atom columns accurately.

Phase Interactions and Focal Series Reconstruction: By measuring lens parameters and recording a series of images at known values of defocus, it is possible to apply a parabolic reconstruction method to generate an estimate of the ESW to the resolution of the highest spatial frequency recorded in the images [17]. This first estimate can be improved by using maximum likelihood to refine the estimates of the experimental parameters [28-29]. The relative phase of the ESW depends linearly on specimen thickness (the number of atoms stacked up in the beam direction) and its spatial distribution reveals the positions of the atom columns. In addition, once the complex ESW has been determined, it is possible to produce any desired image of the experimental specimen with an appropriate "lens function" in the computer [30]. The "phase histogram method" can determine values of defocus and astigmatism automatically from the 
exit-plane wave function, allowing aberrations to be measured and corrected as a spatial function of position in the image [31]. Information about relative atom column positions and weights is present in the same form in both the reconstructed ESW (where it occurs as $\sigma \phi_{\mathrm{p}}(\mathbf{x}) \mathrm{H}$ in the relative phase of the ESW) and in the Scherzer image (where it takes the form $1-2 \sigma \phi_{\mathrm{p}}(\mathbf{x}) \mathrm{H}$ in the image intensity). Thus Scherzer imaging and ESW phase at the same resolution provide the same information about the phase of the structure factors, and hence the positions and weights of atom columns [32].

Focal-series reconstruction (FSR) can be used to determine the ESW to much higher spatial frequencies (better resolution) than the Scherzer image from an uncorrected HRTEM. FSR acts as a "software aberration corrector" on spatial frequencies out to the microscope information limit, improving the microscope resolution to this limit [33]. As well as resolution improvement, another advantage of focal-series reconstruction is that the specimen can be thicker, since it need only satisfy "phase object" conditions instead of the "weak phase object" condition required for Scherzer imaging. Reduced second-order "non-linear" components in the reconstructed phase image allow for a thicker specimen and a "cleaner" result compared with a Scherzer image.

\section{Sub-Ångström atomic-resolution imaging}

\section{a) Microscope Resolution Test Objects}

Silicon and other diamond-cubic and zincblende structures provide useful test specimens for characterization of electron microscope resolution. In [112n] orientation these structures present their atom columns in pairs that have become known as "dumbbells". These arrangements of atoms are advantageous in test specimens because they reduce the "Fourier image" effects that occur with simpler projections, such as in the [100] zone [34-36]. 
Resolution can be determined for both TEM and STEM using the same criterion - separation of atom peaks in the image. A set of physically stable specimens with well-characterized lattice parameters ranging from $6.48 \AA$ for CdTe to $3.56 \AA$ for diamond gives a suitable range of dumbbell atom separations [37]. For the first four zone-axis orientations the test structures cover overlapping regions that allow testing of microscope resolutions from $1.6 \AA$ to $0.2 \AA$ (fig.1).

Models of silicon in the first four orientations ([112n] for $n=0$ to 3 ) show the typical dumbbell arrangement, with atoms in the dumbbell pairs spaced at one quarter of the repeat distance of the dumbbells (fig.2). Increasing the value of $\mathrm{n}$ from 0 to 3 moves the atoms closer together in projection; atom-atom spacing in silicon changes from $1.36 \AA$ for [110] orientation to $0.31 \AA$ for [116] orientation. The models illustrate how atom overlap can impact higher resolutions due to the size of the atoms (here the ball diameter is set to the covalent radius of the atom, or $1.11 \AA$ ). In an electron microscope image, the width of the intensity peak generated from the corresponding atom potential will depend on several factors, including the resolution of the microscope and the spread function of the detector (the resolution of the detection system). In addition, the specimen itself will present atoms of finite "size" to the electron beam [38].

The spatial distribution of projected potential is carried by the relative phase of the scattered electron wave and can be imaged directly to reveal the relative atom positions (to the Scherzer resolution of the microscope) by the interference of correctly phased diffracted beams. Alternatively, the exit-surface wave can be reconstructed and the projected potential (and relative atom positions) imaged by extracting the relative phase. In both cases, resolution in the image can be no better than the "resolution" of the specimen potential. If individual peaks are not separated in the projected potential they will not be separated in the image. 
The four projected potentials computed for the four chosen orientations of silicon conform to the atomic arrangement of the models, with atom columns visible in pairs of peaks forming dumbbells (fig.3). Potential peaks representing the atom columns have a finite size or peak width that depends on the scattering factor for the atoms in the peak - silicon in this case. It is clear that decreasing atom separation will eventually make it impossible to distinguish the individual atom peaks in the potential, let alone in the image.

A major contributor to atom peak width in the projected potential is the Debye-Waller factor. For higher values of this factor, the width of the potential peak (the apparent "size" of the atom) can increase to the point at which smaller atom separations are not resolvable by the electron microscope - even if the instrumental resolution of the microscope is finer. This point can be illustrated by examining the projected potential of silicon in the [116] zone-axis orientation, where the atom columns are only $0.31 \AA ̊$ apart in projection. Increasing the Debye-Waller factor from $0.2 \AA^{2}$ to $1.0 \AA^{2}$ shows how the increasing peak width broadens the individual atom columns until they can no longer be separated in the projected potential (fig.4).

The Debye-Waller factor for silicon has been measured experimentally as $\mathrm{B}=0.4668 \AA^{2}$ at room temperature [39]. Profiles across the atomic potential peaks show that this value still allows separation of the peaks in the dumbbell pair at $0.31 \AA$ (fig.5). In the case of diamond, the Debye-Waller factor is less than half that of silicon, so this limitation will occur at much finer resolutions. Of course, even for a microscope of resolution sufficient to separate ideal potential peaks, additional factors such as specimen tilt can reduce peak separation in the projected potential [40-41]. 


\section{b) Experimental Resolution}

A specimen of germanium tilted into [110] orientation was imaged using a JEOL 2200FS-AC in STEM mode. Although the image is noisy (fig.6), it shows atom peaks in dumbbell pairs. Profiles across the peaks show that the atom peaks are clearly separated by one quarter of the dumbbell repeat and demonstrate $1.41 \AA ̊$ resolution.

For sub-Ångström resolution in TEM mode, a mid-voltage electron microscope needs either a $\mathrm{C}_{\mathrm{S}}$ corrector or some other means of correcting the phase changes introduced by the objective lens at spatial frequencies higher than the Scherzer limit. In addition, the microscope needs to have a sub-Ångström information limit. The information limit is a function of the microscope's spread of focus (eqn. 11) and is strongly dependent on the total energy spread in the incident electron beam (eqn. 12). To keep energy spread low, we require a microscope with a field-emission electron source. Such a microscope can be used to produce sub-Ångström resolution by phase correction (either with holography or by focal-series reconstruction). The One-Ångström Microscope (OAM) at Berkeley uses a combination of focal-series reconstruction software with a highly stable field emission TEM [43].

The OAM hardware is a CM300FEG/UT with lens current and high-voltage power supply stabilities improved from the original values of one part per million. With the original power supply stabilities of one part per million (root mean square), the spread of focus is $36 \AA$, giving an information limit of 1.07 [44]. The CM300FEG/UT-OAM uses improved power supplies with better stabilities to achieve beam energy spread of $0.85 \mathrm{eV} \mathrm{FWHH} \mathrm{[26],} \mathrm{spread} \mathrm{of} \mathrm{focus} \mathrm{of}$ $20 \AA$, and information limit of $0.78 \AA$ [33]. For aberration correction, the OAM uses FEI TrueImage $^{\circledR}$ focal-series reconstruction code [28-29] to compute an estimate of the sample ESW from a focal series of ten to twenty images. Resolution in the computed ESW is limited only by 
the highest spatial frequency contained in the images of the focal series. Under proper conditions, the images can contain spacings out to the information limit of the microscope [45]. The desired result is the phase of the ESW, since the phase is proportional to a projection of the specimen structure [20].

Initial resolution tests of the OAM showed transfer of diffractogram peaks from Au out as far as $0.86 \AA$ [33]. As a test for sub-Ångström resolution we selected a diamond [110] specimen with atom-atom separation of $0.89 \AA$ in the dumbbells (fig.1).

After hardware correction of the OÅM's three-fold astigmatism [46], a "single-shot" image of [110] diamond, obtained close to alpha-null defocus to ensure optimum 004 transfer [47], showed the expected symmetry (fig.7a). A focal series of twenty diamond images was obtained over a range of focus near Lichte defocus [18]. Reconstruction to the exit-surface wave using the Philips/Brite-Euram software [28-29] gave an ESW with a phase that also showed the expected dumbbells (fig.7b). The dumbbell peaks are smoother and clearer than those of the "single-shot" image [33]. Profiling of the two images revealed that the C-C "dumbbell" peaks are spaced slightly closer than one quarter of the dumbbell repeat (fig.7c,d).

With a new HT tank installed, the electron beam energy spread of the OAM (including HT instabilities) was measured as $0.85 \pm 0.05 \mathrm{eV}$ with a Gatan image filter (GIF) at a gun extraction voltage of $3.5 \mathrm{kV}$. Correction for GIF contributions produced a beam energy spread of $0.76 \pm$ $0.05 \mathrm{eV}$ [26] corresponding to an information limit of $0.73 \AA$ to $0.76 \AA$. The OAM was tested using silicon in [112] orientation for $0.78 \AA$ dumbbells (second line in fig. 1).

At $3.5 \mathrm{kV}$ extraction voltage, alpha-null defocus gave a noisy image (fig.8a) with pairs of white peaks that were often blurred together [48] but sometimes showed clear separation (fig.8c). 
Subsequently, a focal series of 20 images was obtained over a range from alpha-null defocus to Lichte defocus. A focal-series reconstruction of the phase of the exit wave (fig. $8 b$ ) from 19 of the images produced an image with softer peaks showing a faint separation (fig.8d). Since imaging (and reconstruction) conditions are close to the microscope's information limit, the peak separation is weak. However, it is clear that there are two peaks in each dumbbell.

\section{Discussion}

\section{a) Quality of Microscope Resolution}

Our dumbbell images of germanium, carbon and silicon (figs.6-8) illustrate differences in the "quality of resolution". Clearly, the atoms in the dumbbells have been "resolved" in all five images, at least in the sense that it is recognizable that there are two atoms present. Two peaks are considered resolved by the Rayleigh criterion [1] when the minimum between them is less than $75 \%$ of the peak height. The Sparrow resolution criterion [49] considers two peaks resolved even when there is no dip between them, as long as their combined curves show a flat maximum; peaks at Sparrow resolution would appear like the curve for $\mathrm{B}=1.0 \AA^{2}$ in figure 4 .

For electron microscopy the important property of improved resolution is the ability to determine the positions of atom columns from image peaks. But peaks in the twin peak may not correspond exactly to the positions of the centers of the individual component peaks [37-38]. At sub-Rayleigh resolutions lying between the Rayleigh and Sparrow criteria image peaks are closer together than atom positions [50]. Determination of individual peak positions requires fitting of computed peaks to those in the experimental image in order to gauge atom positions accurately [37]. A good estimate can be obtained from curves computed over a range of peak separations [50]. A Rayleigh-Sparrow parameter can be defined to describe the quality of resolution in an 
image. This parameter $Q_{R S}=\left(d_{X}-d_{S}\right) /\left(d_{R}-d_{S}\right)$ is equal to one when the atom spacing $d_{X}$ corresponds to Rayleigh spacing $d_{R}$ and to zero when atoms are at Sparrow spacing $d_{S}$. Another useful parameter is $\mathrm{P}_{\mathrm{TP}}$, the ratio of the twin-peak separation in the image to the separation of the individual atom peaks that add to give the twin peaks (or dumbbell).

Experimentally, $\mathrm{Q}_{\mathrm{RS}}$ and $\mathrm{P}_{\mathrm{TP}}$ values can be obtained from the depth of the dip between the twinimage peaks. The variation of $\mathrm{Q}_{\mathrm{RS}}$ and $\mathrm{P}_{\mathrm{TP}}$ with central dip value was measured from twin peaks generated by the addition of two model peaks over a range of separations from Rayleigh to Sparrow (fig.9). From these curves it should be possible to obtain values for resolution quality and twin-peak separation ratio for experimental images by measuring the dip depth. The atom positions are then determined by dividing the observed twin-peak separation by the $\mathrm{P}_{\mathrm{TP}}$ value.

For dumbbell images the separation of the atoms generating the twin peaks is known to be one quarter of the repeat distance of the dumbbell motif, so it is possible to determine the PTP from experimental images and compare it with the predicted value (top line in fig.9). Measured values of dip depth and $\mathrm{P}_{\mathrm{TP}}$ are marked as points on figure 9.

Measurements from the STEM Ge dumbbell image (fig.6) shows that the peaks almost satisfy the Rayleigh resolution criterion with a $\mathrm{Q}_{\mathrm{RS}}$ factor of 0.88 and a $\mathrm{P}_{\mathrm{TP}}$ of $98 \%$ (Table I). Atom separation is $2 \%$ larger, so atom positions lie only $1 \%$ (each) outside the image peak positions.

OÅM diamond dumbbell images (fig.7) are close to Sparrow resolution with $\mathrm{Q}_{\mathrm{RS}}$ and $\mathrm{P}_{\mathrm{TP}}$ factors close to 0.4 and $85 \%$. Measured peak positions are each $7.5 \%$ inside the atom positions.

The OÅM Si [112] dumbbells provide interesting examples (fig.8). The noisy alpha-null image (fig.8a) has a $15 \%$ dip (Table I) so $\mathrm{P}_{\mathrm{TP}}$ is over $90 \%$. The reconstructed phase (fig. $8 \mathrm{~b}$ ) has a dip of less than $2 \%$ making it very close to Sparrow resolution with a $\mathrm{P}_{\mathrm{TP}}$ less than $70 \%$. 


\section{b) Quality of Specimen "Resolution"}

Analysis of the projected crystal potential for silicon columns separated by $0.31 \AA$ (fig.4) reveals that the size of the atom (the Fourier transform of the scattering factor), especially when combined with the Debye-Waller factor, produces dumbbells in the specimen potential in which the twin peaks fail to satisfy the Rayleigh criterion (fig.5). This means that the specimen "object" presented to the scattering electrons may have potential peaks displaced from the positions of atomic columns. For a Debye-Waller factor of $0.4 \AA^{2}$, the twin peaks will be separated by only $87 \%$ of the silicon atom column spacing (Table I), or $0.27 \AA$ instead of $0.31 \AA$.

No increase in microscope resolution will be capable of accurate imaging of atom positions in orientations where the atoms (or atom columns) are too large to allow Rayleigh separation of atom potentials.

\section{Concluding remarks}

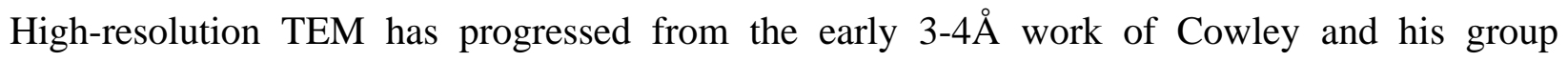
(notably Iijima) to the point of sub-Ångström resolution. At current resolution levels of $0.8 \AA$, the limit to resolution is still instrumental. Limits arising from the Debye-Waller "spreading" of atoms may ultimately occur (requiring cold stages for extreme resolution?), but only when instrumental resolution has improved substantially.

Improved resolution facilitates the imaging of atoms that are close together, but an additional benefit of improved resolution is the ability to image lighter atoms. Focal series reconstruction has shown oxygen atoms at $1.38 \AA$ resolution in staurolite images [51-52]. Oxygen atoms also appear at similar resolutions in images of $\mathrm{ZrO}_{2}$ obtained at $1.3 \mathrm{MeV}$ [53], in focal-series 
reconstructions of $\mathrm{BaTiO}_{3}$ [54], and in $\mathrm{C}_{\mathrm{S}}$-corrected images of perovskites [55]. Reconstruction at sub-Ångström resolution has produced images showing other light atoms, including carbon [33], nitrogen [56], and lithium [57-58]. With HRTEM [48] and STEM [59] images reaching the sub- $0.8 \AA$ level of resolution, it is essential to monitor the quality of resolution to ensure that accurate atom positions can be determined.

Given its wide range of detectable atoms, the HRTEM is confirmed in its position as the preferred instrument for the imaging of atoms. The emergent nanotechnology revolution requires knowledge of atom positions to relate structure to properties, and HR-(S)TEM at subÅngström resolution will play an essential role. Our early reconstruction results foreshadow those to be expected from emerging TEMs with $\mathrm{C}_{\mathrm{S}}$-corrected lenses and monochromated electron beams.

\section{Acknowledgments}

The first author is grateful to Professor Cowley for his guidance on the road that has led to the achievement of sub-Ångström resolution and the imaging of light atoms, and to Dr. David J. Smith for critical comments on the manuscript. This work was supported by the Director, Office of Science, Office of Basic Energy Sciences, Materials Sciences Division, of the U.S. Department of Energy, under contract No. DE-AC03-76SF00098 and the Assistant Secretary for EERE, Office of FreedomCAR and Vehicle Technology, HTML User Program, ORNL, managed by UT-Battelle, LLC for DOE (contract DE-AC05-00OR22725). Electron microscopy was performed at the Advanced Microscopy Laboratory at Oak Ridge National Laboratory, and at the National Center for Electron Microscopy at Lawrence Berkeley National Laboratory. 


\section{REFERENCES}

1. Strutt J W (Lord Rayleigh) (1874). On the manufacture and theory of diffraction gratings. Philosophical Magazine 47, 310: 81-93.

2. den Dekker A J and van den Bos A (1997) Resolution: a survey. Journal of the Optical Society of America A 14: (3) 547-557.

3. Allpress J G (1969) The direct observation of structural features and defects in complex oxides by two-dimensional lattice imaging. Mat. Res. Bull. 310: 707-712.

4. Iijima S (1971) High-resolution electron microscopy of crystal lattice of titanium-niobium oxide. J. Appl. Phys. 42: 5891-5893.

5. Cook J M., O'Keefe M A, Smith D J and Stobbs W M (1983) The high resolution electron microscopy of stacking defects in Cu-Zn-Al shape-memory alloy. J. Microscopy 129: 295-306.

6. Epicier T, O'Keefe M A and Thomas G (1990) Atomic imaging of 3:2 mullite. Acta Cryst. A46: 948-962.

7. Allpress J G, Hewat E A, Moodie A F and Sanders J V (1972) n-beam lattice images, I. Experimental and computed images from $\mathrm{W}_{4} \mathrm{Nb}_{26} \mathrm{O}_{77}$. Acta Cryst. A28: 528-535.

8. Lynch D F and O'Keefe M A (1972) n-beam lattice images, II. Methods of calculation. Acta Cryst. A28: 536-548.

9. Anstis G R, Lynch D F, Moodie A F and O'Keefe M A (1973). n-beam lattice images, III. Upper limits of ionicity in $\mathrm{W}_{4} \mathrm{Nb}_{26} \mathrm{O}_{77}$. Acta Cryst. A29: 138-147.

10. O'Keefe M A (1973) n-beam lattice images, IV. Computed two-dimensional images. Acta Cryst. A29: 389-401. 
11. Lynch D F, Moodie A F and O'Keefe M A (1975) n-beam lattice images, V. Use of the charge-density approximation in the interpretation of lattice images. Acta Cryst. A31: 300-307.

12. O'Keefe M A and Sanders J V (1975) n-beam lattice images, VI. Degradation of image resolution by a combination of incident-beam divergence and spherical aberration. Acta Cryst. A31: 307-310.

13. O'Keefe M A, Buseck P R and Iijima S (1978). Computed crystal structure images for highresolution electron microscopy. Nature 274: 322-324.

14. O'Keefe M A, Buseck P R (1979) Computation of high-resolution TEM images of minerals. Transactions of the ACA 15: 27-46.

15. Scherzer O (1949). The theoretical resolution limit of the electron microscope. J. Applied Physics, 20: 20-29.

16. Schiske P (1973) in: Image Processing And Computer-Aided Design, ed. Hawkes P W, pp.8290, (Acad. Press, London).

17. Van Dyck D. and Op de Beeck M. (1990) New direct methods for phase and structure retrieval in HREM. Proceedings of the 12th International Congress for Electron Microscopy, ed. Peachy L D and Williams D B, pp. 26-27 (Seattle, Washington).

18. Lichte H (1991) Optimum focus for taking electron holograms. Ultramicroscopy 38: 13-22.

19. Haider M, Braunshausen G. and Schwan E (1995) Correction of the spherical aberration of a 200kV TEM by means of a hexapole-corrector. Optik, 99: 167-179.

20. Cowley J M and Iijima S (1972) Electron microscope image contrast for thin crystals. $Z$. Naturforsch. 27a: 445-451. 
21. O'Keefe M A (1992). "Resolution" in high-resolution electron microscopy. Ultramicroscopy 47: 282-297.

22. O'Keefe M A (1979). Resolution-damping functions in non-linear images. 37th Ann. Proc. EMSA, San Antonio, Texas 556-557.

23. Cowley J M and Moodie A F (1957) The scattering of electrons by atoms and crystals. I. A new theoretical approach. Acta Cryst. 10: 609-623.

24. Frank J (1973) The envelope of electron microscopic transfer functions for partially coherent illumination. Optik 38: 519-536.

25. Wade R H and Frank J (1977) Electron microscope transfer functions for partially coherent axial illumination and chromatic defocus spread. Optik 49: 81-92.

26. O'Keefe M A, Tiemeijer P C and Sidorov M V (2002) Estimation of the electron beam energy spread for TEM information limit. Microscopy \& Microanalysis 8, 2: 480-481.

27. Malm J-O and O'Keefe M A (1993) Using convergence and spread-of-focus parameters to model spatial and temporal coherence in HRTEM image simulations. 51st Ann Proc. MSA, Cincinnati, Ohio, 974-975.

28. Coene W M J, Thust A, Op de Beeck M. and Van Dyck D (1996) Maximum-likelihood method for focus-variation image reconstruction in high-resolution transmission electron microscopy. Ultramicroscopy 64: 109-135.

29. Thust A, Coene W M J, Op de Beeck M and Van Dyck D (1996) Focal-series reconstruction in HRTEM: simulation studies on non-periodic objects. Ultramicroscopy, 64: 211-230.

30. Thust A, Overwijk M, Coene W and Lentzen M (1996) Numerical correction of lens aberrations in phase-retrieval HRTEM. Ultramicroscopy, 64: 249-260. 
31. Thust A, Overwijk M, Jia C L and Urban K (2003) Direct determination of imaging parameters from wave functions in HRTEM. Microsc. Microanal. 9, Suppl. 2: 140-142.

32. O'Keefe M A (2004) Image formation in the high-resolution transmission electron microscope, Microscopy \& Microanalysis 10: 397-399.

33. O'Keefe M A, Hetherington C J D, Wang Y C, Nelson E C, Turner J H, Kisielowski C, Malm J-O, Mueller R, Ringnalda J, Pan M and Thust A (2001). Sub-Ångström high-resolution transmission electron microscopy at 300keV. Ultramicroscopy 89, 4: 215-241.

34. Cowley J M and Moodie A F (1957) Fourier images. I. The point source. Proc. Phys. Soc. B 70: $486-496$.

35. Iijima S and O'Keefe M A (1979) Determination of defocus values using 'Fourier images' for high-resolution electron microscopy. J. Micros. 117: 347-354.

36. O'Keefe M A, Dahmen U and Hetherington C J D (1989) Simulated image maps for use in Experimental High-Resolution Electron Microscopy, Mat. Res. Soc. Symp. Proc. 159: 453-458.

37. O'Keefe M A and Allard L F (2004) A standard for sub-Ångström metrology of resolution in aberration-corrected transmission electron microscopes. Microscopy \& Microanalysis 10: 10021003.

38. Van Dyck D, Van Aert S and den Dekker A J (2004). Physical limits on atomic resolution. Microscopy \& Microanalysis 10: 153-157.

39. Zuo J M, Blaha P and Schwarz K (1997) The theoretical charge density of silicon: experimental testing of exchange and correlation potentials. J. Phys.: Condens. Matter 9: 75417561. 
40. O'Keefe M A and Radmilovic V (1993) The effects of small crystal tilts on dynamical scattering: why simulated images are thinner than experimental ones. 51st Ann Proc. MSA, Cincinnati, Ohio, 980-981.

41. O'Keefe M A and Radmilovic V (1994). Specimen thickness is wrong in simulated HRTEM images. Proc. XIIIth Int. Congress for Electron Microscopy 1, 361-362.

42. Allard L F, Blom D A, O'Keefe M A, Kiely C, Ackland D, Watanabe M, Kawasaki M, Kaneyama T and Sawada H (2004) First Results from the Aberration-Corrected JEOL 2200FSAC STEM/TEM. Microscopy \& Microanalysis 10: 110-111.

43. O'Keefe M A (1993) Using coherent illumination to extend HRTEM resolution: why we need a FEG-TEM for HREM. In: LBL Symposium on Microstructures of Materials, ed by Krishnan K, pp. 121-126, (Berkeley: San Francisco Press).

44. Bakker H, Bleeker A and Mul P (1996) Design and performance of an ultra-high-resolution $300 \mathrm{kV}$ microscope. Ultramicroscopy 64: 17-34.

45. O'Keefe M A (2001) Alpha-null defocus: an optimum defocus condition with relevance for focal-series reconstruction. Microscopy \& Microanalysis 7, 2: 916-917.

46. Typke D and Dierksen K (1995) Determination of image aberrations in high-resolution electron microscopy using diffractogram and cross-correlation methods. Optik 99, 4: 155-166.

47. Wang Y C, Fitzgerald A, Nelson E C, Song C, O'Keefe M A and Kisielowski C (1999) Effect of correction of the 3-fold astigmatism on HREM lattice imaging with information below $100 \mathrm{pm}$. Microscopy \& Microanalysis 6, 2: 822-823.

48. O'Keefe M A, Nelson E C, Wang Y C and Thust A (2001) Sub-Ångström resolution of atomistic structures below 0.8̊̊. Philosophical Magazine B 81, 11: 1861-1878. 
49. Sparrow C M (1916). On spectroscopic resolving power. Astrophys. Journal 44: 76-86.

50. O'Keefe M A, Allard L F and Blom D A (2005) Resolution quality and atom positions in sub-Ångström electron microscopy, Microscopy \& Microanalysis 11 (2005) accepted.

51. Downing, K.H., Meisheng, Hu, Wenk, H.-R and O'Keefe, M.A. (1990). Resolution of oxygen atoms in staurolite by three-dimensional transmission electron microscopy. Nature 348, 525-528.

52. Wenk H-R, Downing K H., Meisheng Hu and O'Keefe M A (1992) 3d structure determination from electron-microscope images: electron crystallography of staurolite. Acta Cryst. A48: 700716.

53. Horiuchi S, Matsui Y, Kitami Y, Yokoyama M, Suehara S, Wu X J, Matsui I and Katsuta T (1991) Ultra-high-resolution HVEM (H-1500) newly constructed at NIRIM. II. Application to materials. Ultramicroscopy 39: 231-237.

54. Jia C L and Thust A (1999) Investigation of atomic displacements at a $\Sigma 3\{111\}$ twin boundary in $\mathrm{BaTiO}_{3}$ by means of phase-retrieval electron microscopy. Phys. Rev. Letts $\mathbf{8 2}$ : $5052-5055$.

55. Jia C L, Lentzen M and Urban K (2003) Atomic-resolution imaging of oxygen in perovskite ceramics. Science 299: 870-873.

56. Kisielowski C, Hetherington C J D, Wang Y C, Kilaas R, O'Keefe M A and Thust A (2001) Imaging columns of the light elements carbon, nitrogen and oxygen at sub-Ångström resolution. Ultramicroscopy 89: 243-263.

57. Shao-Horn Y, Croguennec L and Delmas C, Nelson E C, O'Keefe, M A (2003). Atomic resolution of lithium ions in $\mathrm{LiCoO}_{2}$. Nature Materials 2: 464-467. 
58. O'Keefe M A and Shao-Horn Y (2004) Sub-Ångström atomic-resolution imaging from heavy atoms to light atoms. Microscopy \& Microanalysis 10: 86-95.

59. Nellist P D, Chisholm M F, Dellby N, Krivanek O L, Murfitt M F, Szilagyi Z S, Lupini A. R, Borisevich A, Sides W H Jr. and Pennycook S J (2004) Direct sub-angstrom imaging of a crystal lattice. Science 305, 5691: 1741-1741. 
Table I - Quality of resolution parameter $\mathrm{Q}_{\mathrm{RS}}$ for the experimental images at sub-Rayleigh resolution. Central dip and twin-peak separation parameter $\mathrm{P}_{\mathrm{TP}}$ measured from experimental images and from silicon potential data computed over a range of Debye-Waller factors.

Image

[110] Ge (fig.6d)

[110] diamond (fig.7c)

[110] diamond (fig.7d)

[112] Si (fig.8c)

[112] Si (fig.8d)

[116] Silicon with D-W $=0.2 \AA^{2}$

[116] Silicon with D-W $=0.4 \AA^{2}$

[116] Silicon with D-W $=0.6 \AA^{2}$

[116] Silicon with D-W $=0.8 \AA^{2}$
$\underline{\mathrm{Q}_{\mathrm{RS}}}$

0.88

0.40

0.42

0.68

0.15

23.5

11.0

5.1

2.0
$\underline{\mathrm{P}}_{\mathrm{TP}}(\%)$

98.0

83.3

86.7

91.2

67.9

96.2

86.7

76.2

57.1 


\section{Figure Captions}

Fig. 1. Atom-atom spacings within dumbbells for seven compounds with diamond cubic or zincblende structure plotted as a function of lattice parameter for the four zone-axis orientations ([1 $112 \mathrm{n}] \mathrm{n}=0,1,2,3)$. Resolutions needed to image atoms within pairs in [110] orientation range from $1.62 \AA$ for $\mathrm{CdTe}$ to $0.89 \AA$ for diamond. Complex orientations (higher values of n) require better resolution. The four orientations cover four overlapping resolution ranges (left) from $1.62 \AA$ to $0.20 \AA$.

Fig. 2. Models of silicon oriented to form "dumbbell" pairs of atoms in $\left[\begin{array}{lll}1 & 1 & 2 n\end{array}\right]$ orientations. Dumbbell spacing decreases as silicon is projected in increasingly complex zone-axis orientations (marked). Vertical repeat is $|\mathbf{a}| / \sqrt{ } 2=3.84 \AA$ in each case. Atom-atom spacing within each dumbbell is $1 / 4$ of the separation between dumbbells. Atoms are drawn as hard spheres with $1.11 \AA$ diameter. Atom-atom spacings within the dumbbells range from $1.36 \AA$ in $[110]$ orientation to $0.31 \AA$ in [116] orientation. Shown at 40MX magnification.

Fig. 3. Silicon crystal potentials projected down four zone axes in $\left[\begin{array}{lll}1 & 1 & 2 n\end{array}\right]$ orientation show dumbbell pairs of atoms in positions corresponding to those in the models. Projected potentials are calculated from structure factors out to $8 \AA-1$. Shown at $40 \mathrm{MX}$ magnification. DebyeWaller temperature factors are 1.0, 0.8, 0.6 and $0.4 \AA 2$.

Fig. 4. Silicon crystal potential projected down the [116] zone axes shows the effect of temperature on the "size" of the silicon atoms. Displays of projected potentials, calculated from structure factors out to $8 \AA-1$, illustrate how atoms in each pair become less distinct as the Debye-Waller factor (shown) increases. 50MX magnification. 
Fig. 5. Profiles of silicon crystal potential projected down the [116] zone axes display the effect of temperature factor (shown) on projected potential. Individual atom peaks in each pair become less distinct as the Debye-Waller factor is increased. Shown at 50MX magnification.

Fig. 6. a. Experimental image of germanium in [110] orientation taken from a large area with $1.41 \AA$ atom-atom spacing in dumbbells. The image was obtained with the ORNL "ACEM", a JEOL 2200FS-AC, operating in STEM HAADF mode with a CS-corrected probe. b. Image after high-frequency noise filtering. c. Profile across raw image. d. Profile across noisefiltered image.

Fig. 7. Experimental images of diamond in [110] orientation show $0.89 \AA$ atom-atom spacing in dumbbells. a. Single-shot image taken near alpha-null defocus shows pairs of carbon atoms. b. OÅM image reconstructed from a 20-member focal series. c. Profile of double peak marked in a shows clear peak separation. d. Profile of atom pair marked in c. Dashed lines in profiles show peak separation; solid lines are spaced at $1 / 4$ of the dumbbell repeat distance.

Fig. 8. Experimental OÅM-CM300 images of silicon in [112] orientation with super-imposed models of silicon. a. Single-shot image taken near alpha-null defocus shows pairs of silicon atoms separated by $0.784 \AA$. b. OÅM image reconstructed from a 19-member focal series. c. Profile of double peak marked in a shows clear $0.784 \AA$ peak separation. d. Profile of atom pair marked in c shows blunt peaks with less separation. Dashed lines in profiles show peak separation; solid lines are spaced at $1 / 4$ of the dumbbell repeat distance.

Fig. 9. Plots generated from model peaks of PTP (black line) and QRS (dashed) as functions of the depth of the twin-peak dip. Black points are measured from experimental images with PTP computed from known atom peak spacing. Error bars represent \pm 0.5 pixel in peak measurement. 


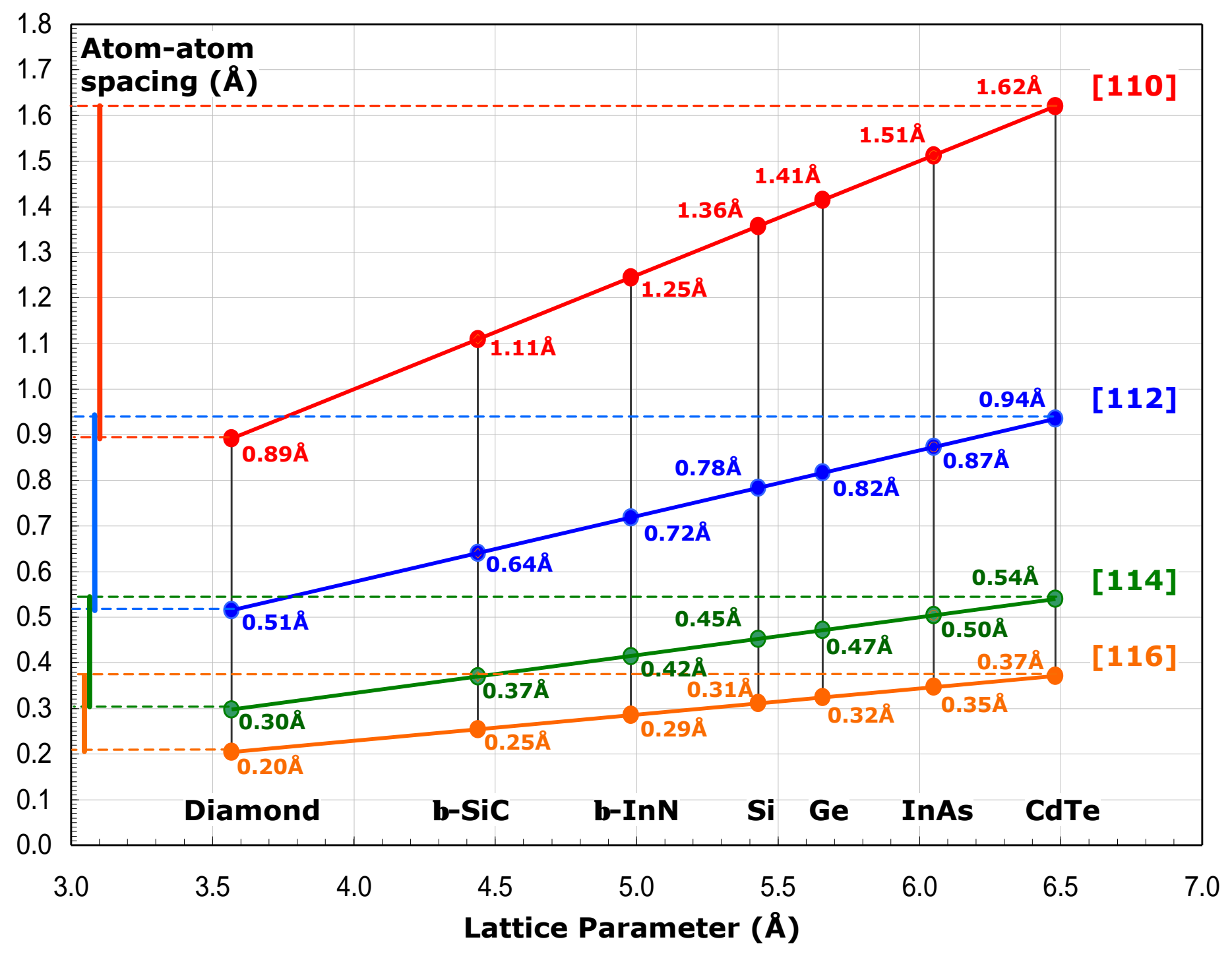

Fig. 1.

Michael A. O'Keefe, Lawrence F. Allard and Douglas A. Blom.

HRTEM Imaging of Atoms at Sub-Ångström Resolution 

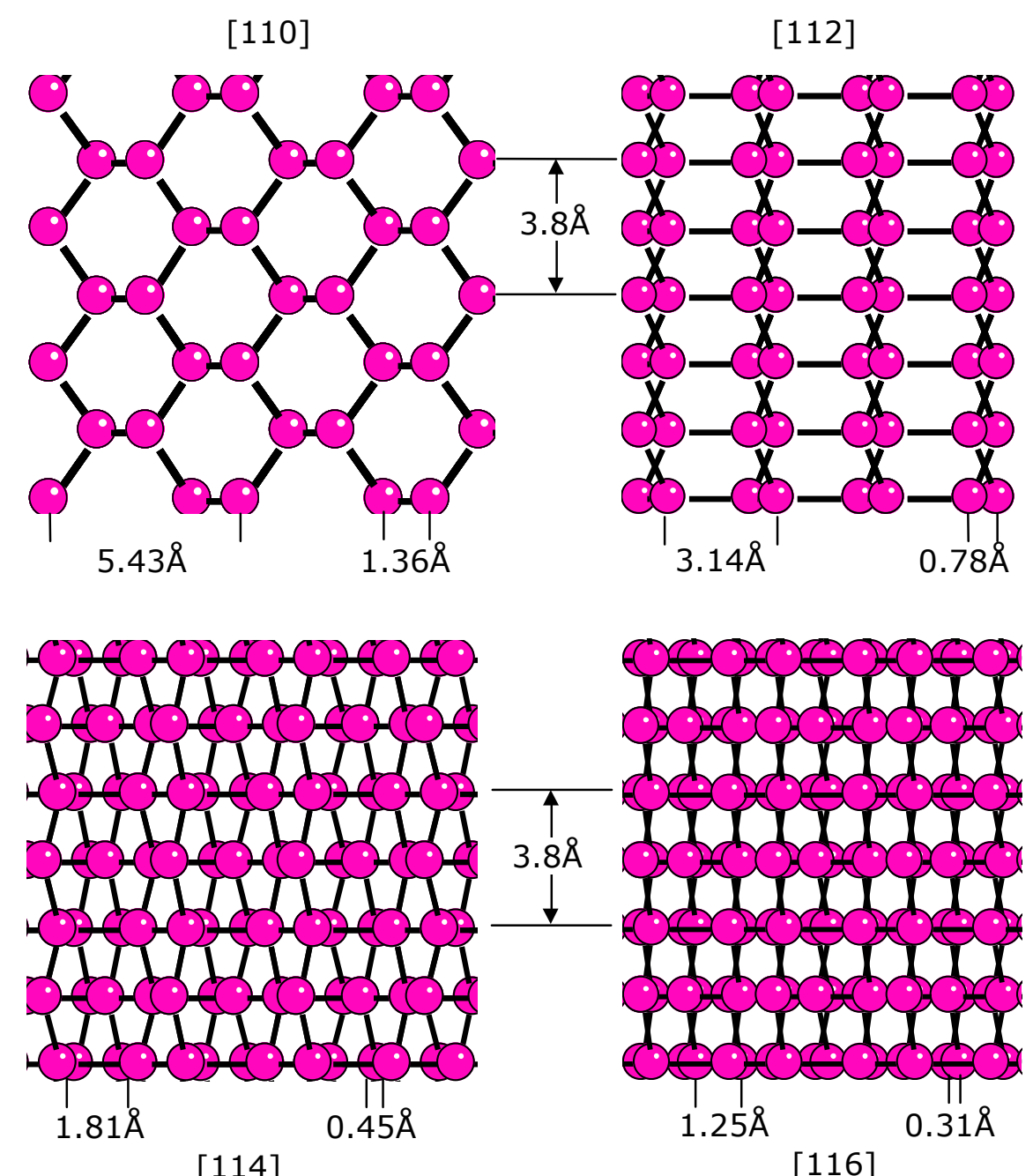

Fig. 2.

Michael A. O'Keefe, Lawrence F. Allard and Douglas A. Blom. HRTEM Imaging of Atoms at Sub-Ångström Resolution 

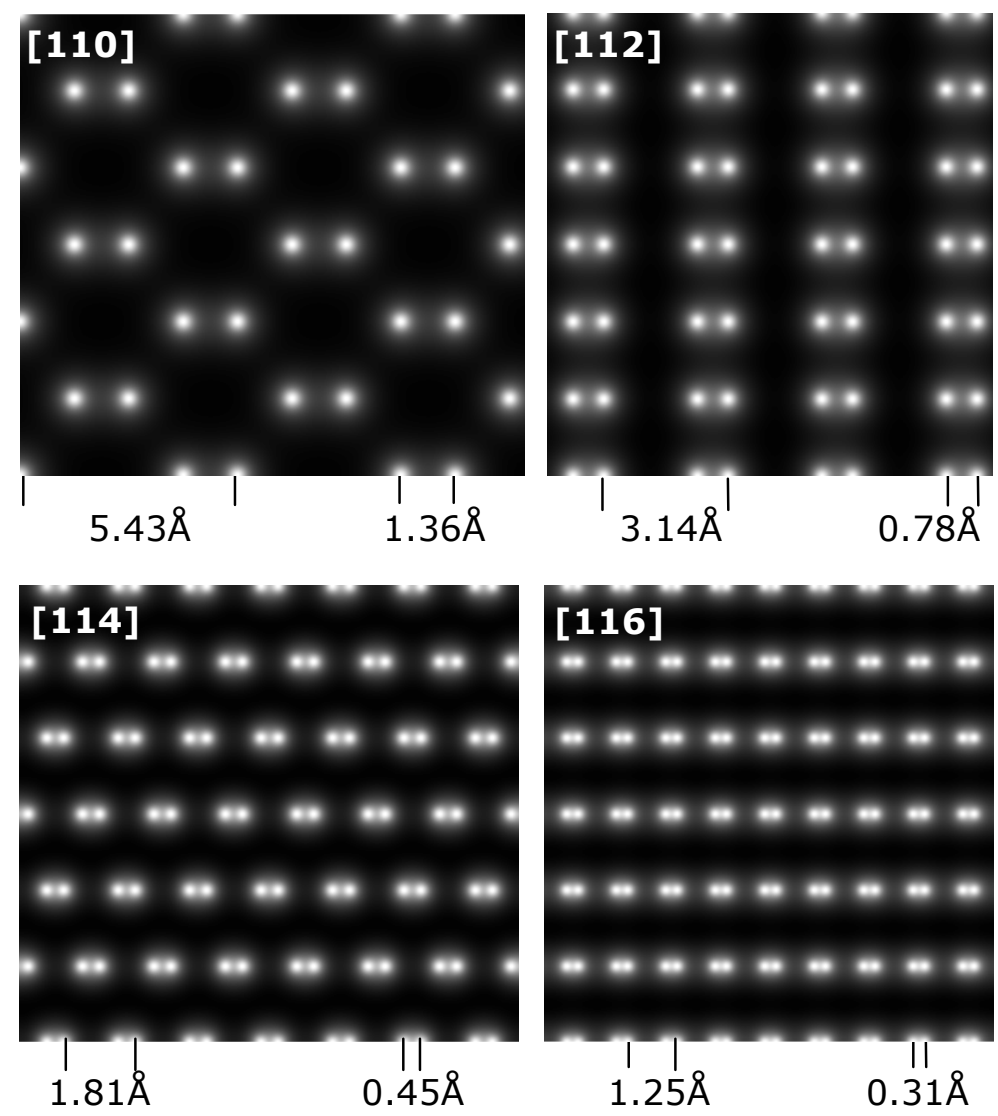

Fig. 3.

Michael A. O'Keefe, Lawrence F. Allard and Douglas A. Blom.

HRTEM Imaging of Atoms at Sub-Ångström Resolution 


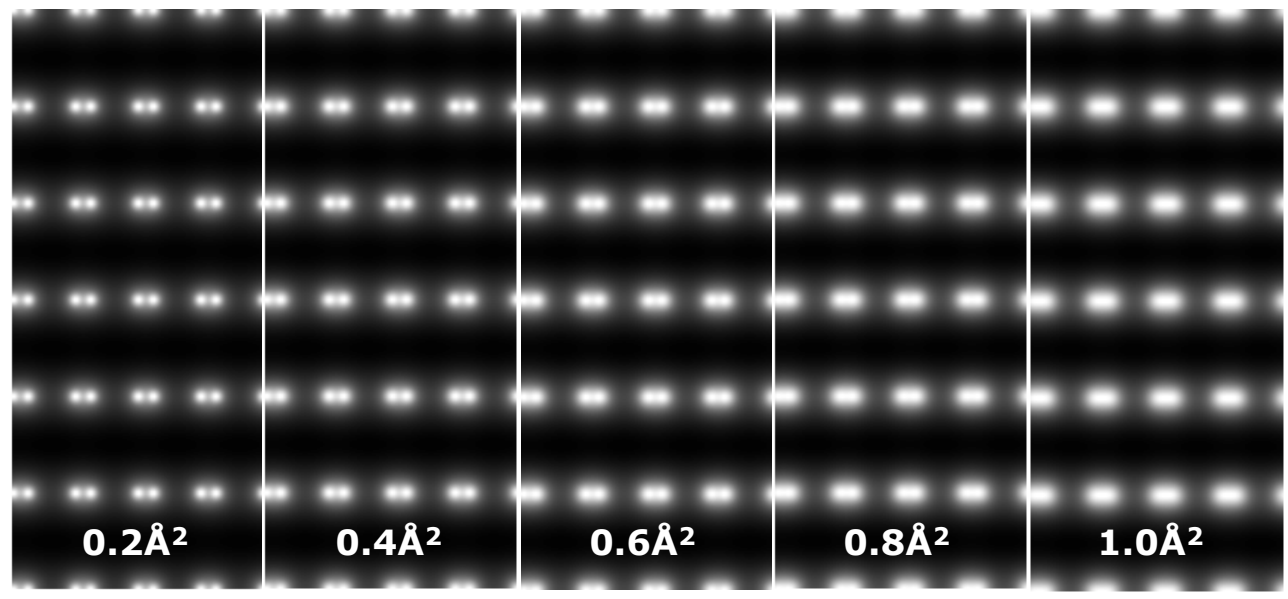

Fig. 4.

Michael A. O'Keefe, Lawrence F. Allard and Douglas A. Blom.

HRTEM Imaging of Atoms at Sub-Ångström Resolution 

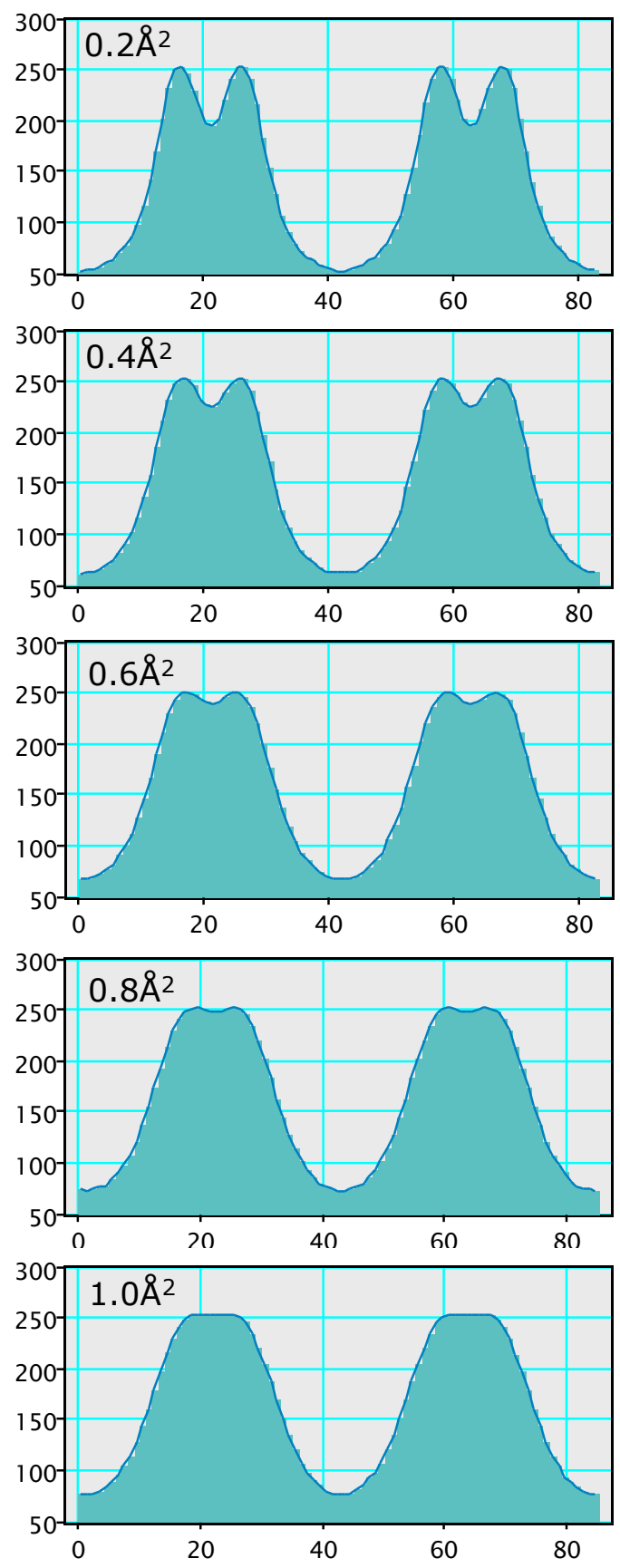

Fig. 5.

Michael A. O'Keefe, Lawrence F. Allard and Douglas A. Blom.

HRTEM Imaging of Atoms at Sub-Ångström Resolution 


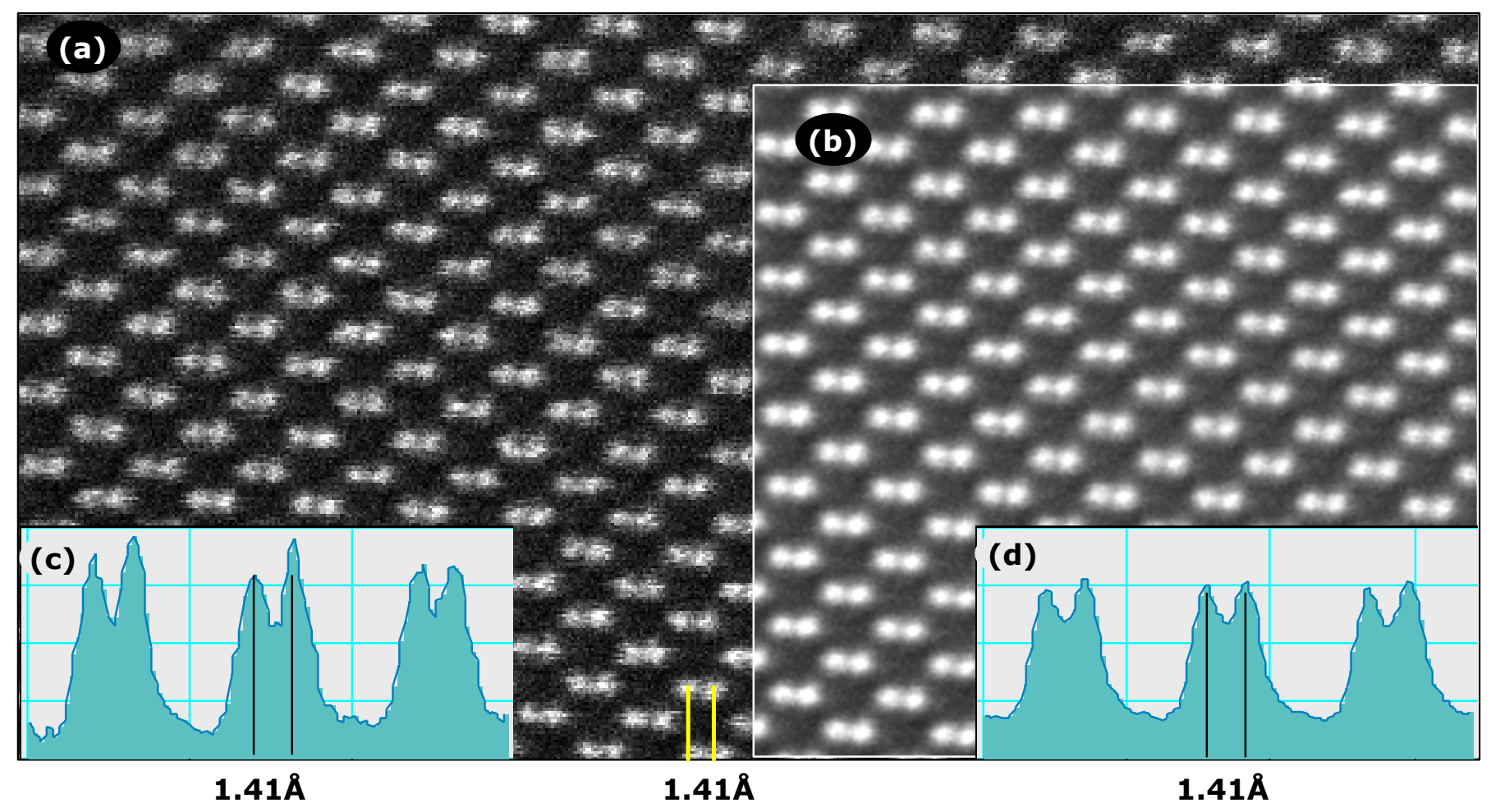

Fig. 6.

Michael A. O'Keefe, Lawrence F. Allard and Douglas A. Blom.

HRTEM Imaging of Atoms at Sub-Ångström Resolution 

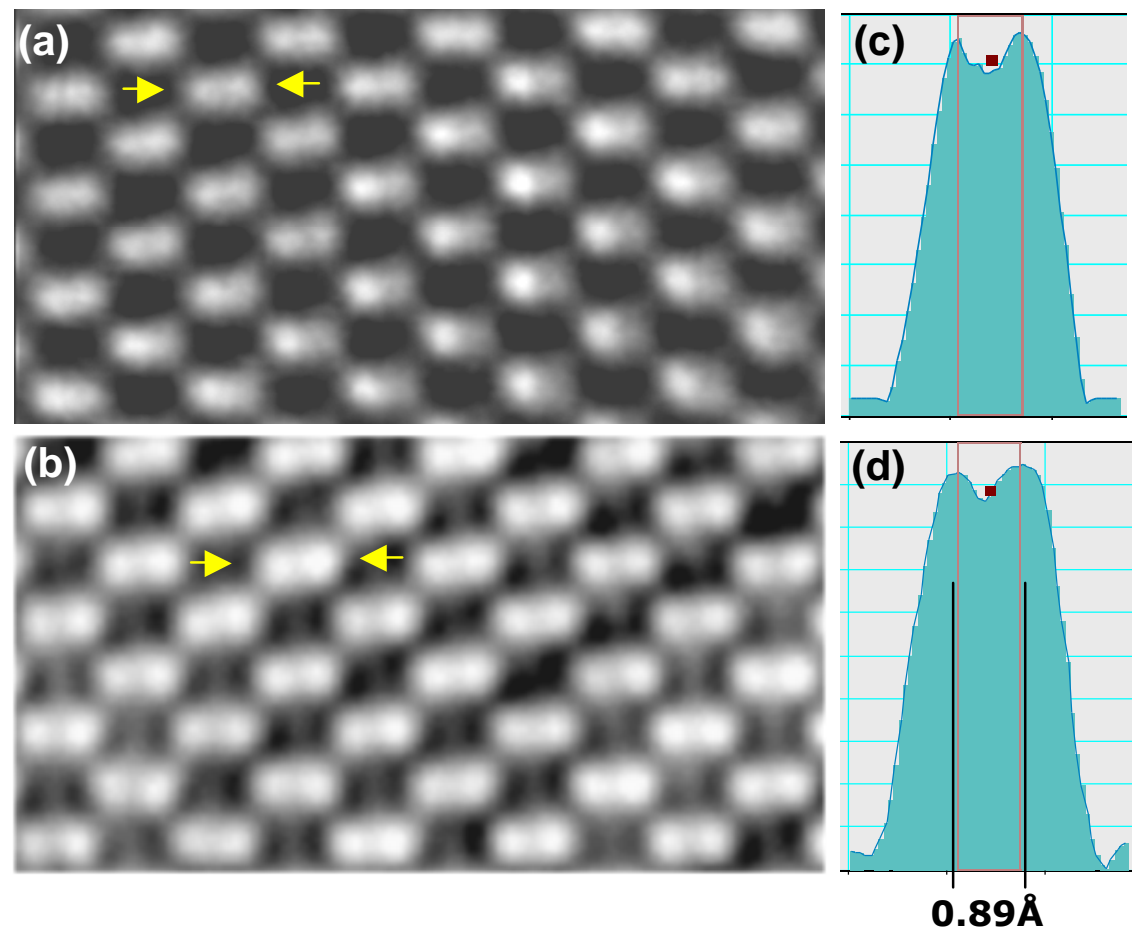

Fig. 7.

Michael A. O'Keefe, Lawrence F. Allard and Douglas A. Blom. HRTEM Imaging of Atoms at Sub-Ångström Resolution 

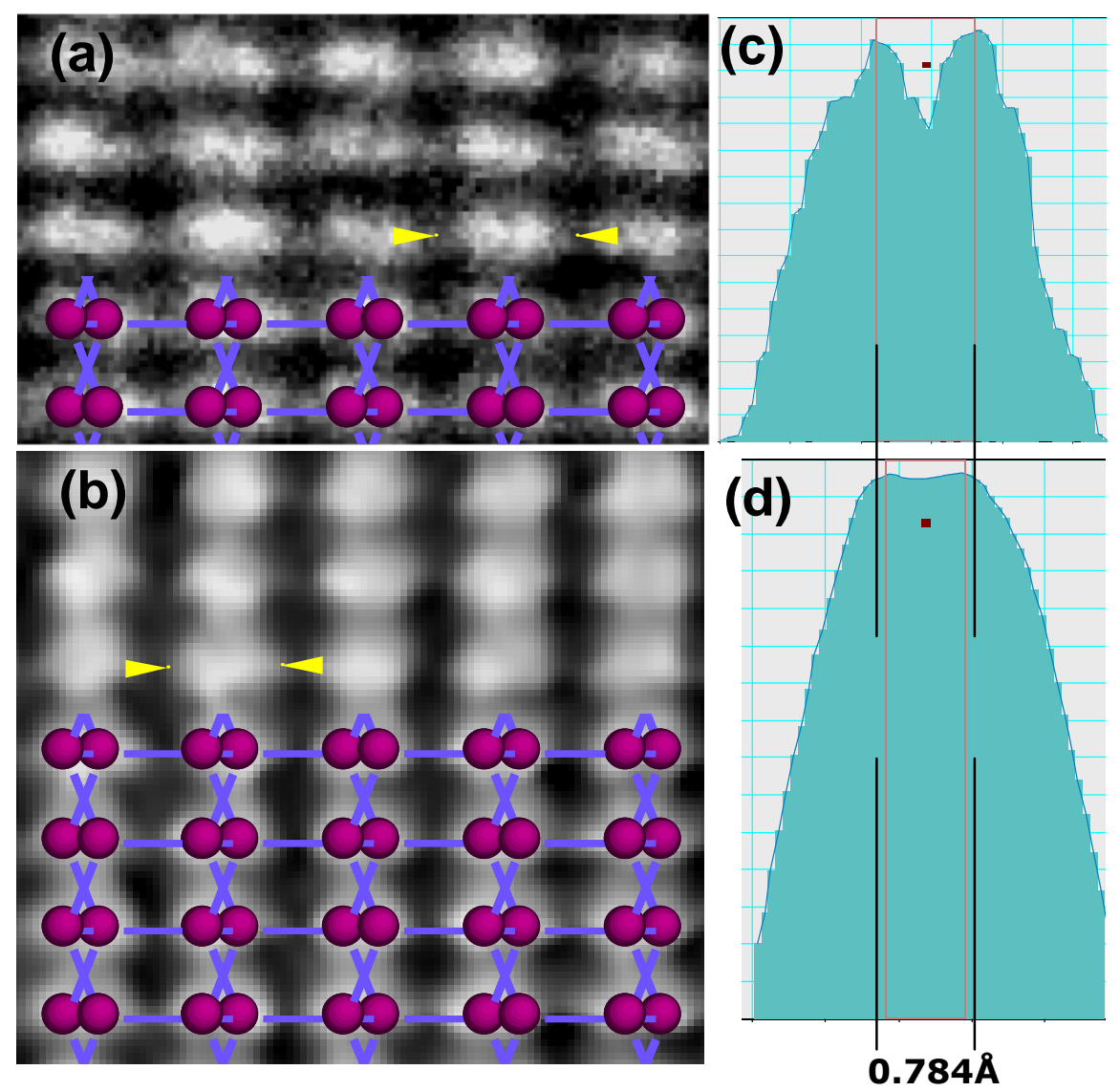

Fig. 8.

Michael A. O'Keefe, Lawrence F. Allard and Douglas A. Blom. HRTEM Imaging of Atoms at Sub-Ångström Resolution 


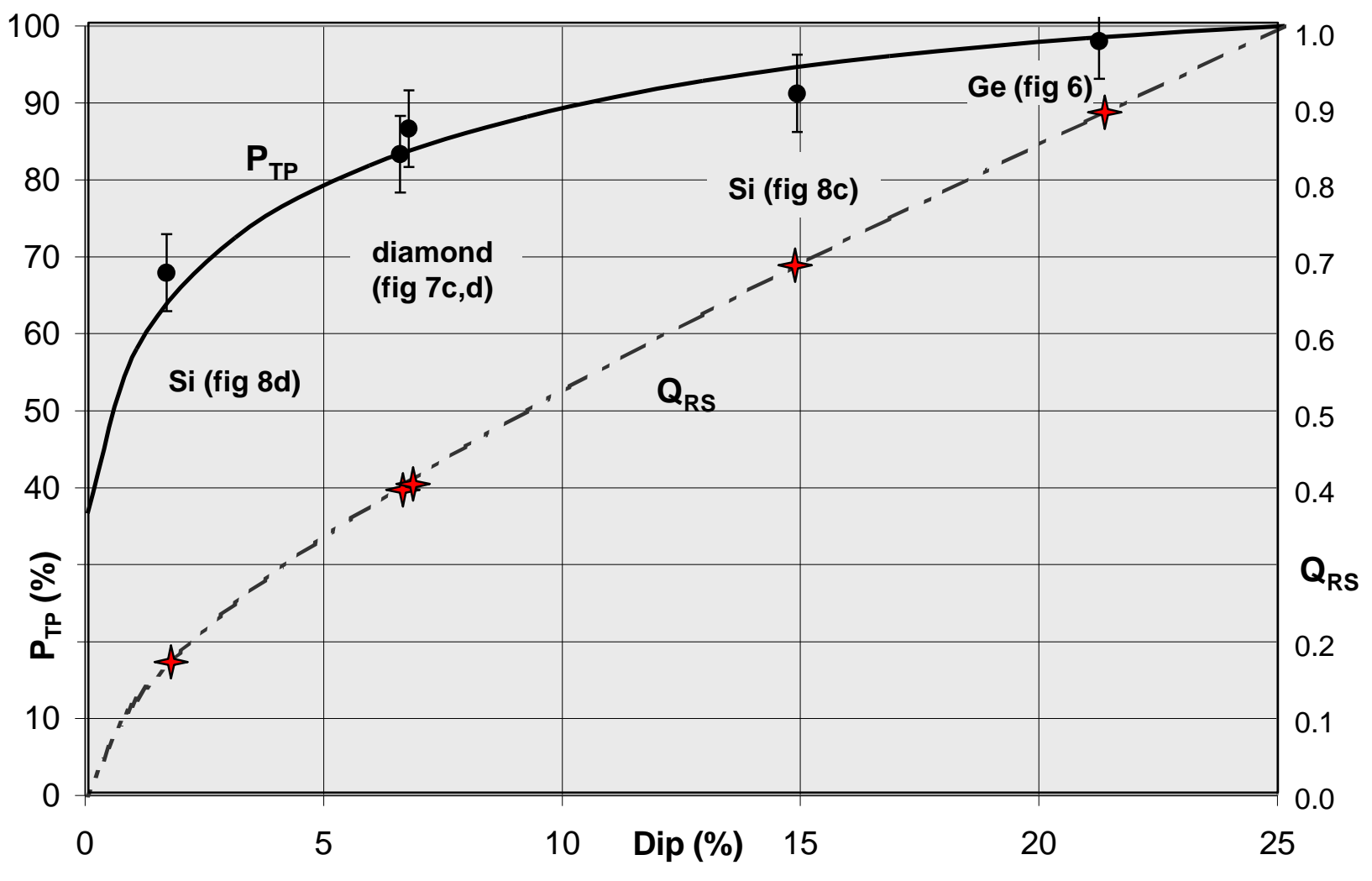

Fig. 9.

Michael A. O'Keefe, Lawrence F. Allard and Douglas A. Blom.

HRTEM Imaging of Atoms at Sub-Ångström Resolution 